\title{
Fungal Laryngitis in Immunocompetent Patients; Risk Factors, Presentation and Treatment
}

\author{
Ibrahim J Issa* and James P Thomas \\ Laryngologist and Phono Surgeons, Soliman Fakeeh Hospital, Saudi Arabia
}

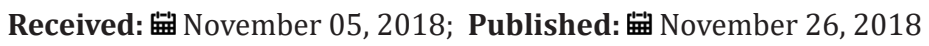

*Corresponding author: Ibrahim J Issa, Laryngologist and Phono Surgeons, Soliman Fakeeh Hospital, Jeddah, Saudi Arabia

\begin{abstract}
Introduction: Fungal laryngitis in immunocompetent patients is an underestimated disease that is not enough studied in the literature. In this study we are presenting the largest patients' charts data analysis up to this date.

Results: Ninety three patients with fungal laryngitis are analyzed in this study. Ninety one of them were presumably immunocompetent. 69 patients (74.19\%) were found to be using inhaled steroids, 15 patients (16.13) were using oral steroids, 8 patients $(8.60 \%)$ were using nasal steroid sprays, 11 patients $(11.83 \%)$ were on antibiotics, 13 patients (13.98) have reflux symptoms. Other risk factors like diabetes mellitus, history of intubation, vocal cord palsy, and smoking were also found and studied. Precise and detailed analysis was done for the presenting appearance of the lesions, most patients were found to present with a thin white plaque like lesions, many patients presented with varying types of lesions ranging from subtle whitish spots with surrounding inflammatory changes to as obvious as ulcer like elevated thick white lesions on the vocal cords.

Discussion: Low threshold for the diagnosis of fungal laryngitis is needed when appreciating one or more of the disease risk factors, detailed high definition video-stroposcopy is needed to diagnose and follow patients' lesions and their progression with treatment. We have divided patients into six groups regarding their presenting lesions appearance, studying each presentation percentage and showing laryngoscopy examples makes it easy to diagnose patients, follow them up and assessing the change in lesion with treatment. Under estimation of the problem common occurrence, lack of awareness of the appearance of these lesions, over estimation of other laryngeal problems, and the inappropriate low definition rapid less detailed laryngoscopic examination are some presumed causes behind missing most of the patients with this diagnosis.
\end{abstract}

\section{Introduction}

Fungal laryngitis is a rare infection of the laryngeal structures by the fungus present in most of the cases as normal flora in the larynx, occurring mostly in immunocompromised patients. Nevertheless, this infection could also happen not uncommonly in immunocompetent patients, a disease that is much underestimated by Laryngologists and Otolaryngologists due to several factors that as we think may include lack of diagnostics precision, over diagnosis of other non-confirmable disorders like subtle Laryngopharyngeal reflux (LPR) as a cause of many voice problems, and most importantly due to lack of awareness of presentation variabilities of this disease. In this study we aim to emphasize on the different presentations of fungal laryngitis in immunocompetent patients, as well as, studying the risk factors associated with this disease, that should make the physician alert to the possibility of this diagnosis when they are found in a patient. Fungal laryngitis in immunocompetent patients is not studied thoroughly in the literature, few studies and case reports have studied this disease and linked the occurrence of this infection to the use of inhaled corticosteroid medications of asthma. In this study we are conducting the largest clinical analysis of patients complaining of fungal laryngitis up to the publication date, trying to find the most important risk factors associated with this infection, the variable presenting patterns and sites, and time needed for treatment of this infection.

\section{Methods}

This is a retrospective chart review analysis, we reviewed all patients diagnosed in James Thomas voice center, Portland, OR with fungal laryngitis between the period of (1999-2016) , we evaluated patients chart for the cases with confirmed diagnosis, analyzing all the aspects of this disease including patients' age, gender, exposure to risk factors suggested in the literature as well as other risk factors suggested by the author, including inhaled steroids, oral steroids, nasal steroids, antibiotic use in the period of infection or two week before, history of acid reflux symptoms, history of diabetes, smoking, history of intubation during the last year before 
diagnosis, history of immunosuppression, history of radiotherapy or chemotherapy during the last year before diagnosis, evidence of any vocal cord abnormality preceding and during the period of infection. Diagnosis of fungal laryngitis was clinical, based on patient's history including the onset of the problem and its close relation with the risk factors combined with inflammatory changes of the vocal cords that represent candida whitish discoloration associated with rapid and obvious improvement on antifungal tablets in subsequent follow up laryngoscopies. Biopsy and swap cultures were not needed for diagnosis due to the resolution of the infection before the expected time of biopsy results and the need for invasive procedure with Potential complications to have them, adding to that, the fact that laryngeal Candida responds extremely well to treatment, which makes the option of a biopsy unappealing We needed to biopsy four cases were the improvement was slow and lesions were more suspicious of a malignant process, and the histopathology result came out confirming the presence of candida element with inflammatory changes [1]. We have also studied the way of presentation those patients presented with, including the site of involvement, symptoms on presentation, and the variation of lesions' appearance, using the records of the patients' examination with high definition laryngoscopes and stroboscope. We evaluated the previous diagnoses patients were labeled with before presenting to us, trying to find the cause of diagnosis delay if present and the other diagnoses physicians are mislabeling patients with, and the cause behind that. Lastly, we calculated the time needed for cure of fungal laryngitis in our patients using our treatment protocol which is based on oral Fluconazole $200 \mathrm{mg}$ for the first day and then $100 \mathrm{mg}$ till complete resolution of the infection), minimize the theoretical risk factors, use of laryngeal gargles after inhaled steroids for patients who need to use them [2-4].

\section{Results}

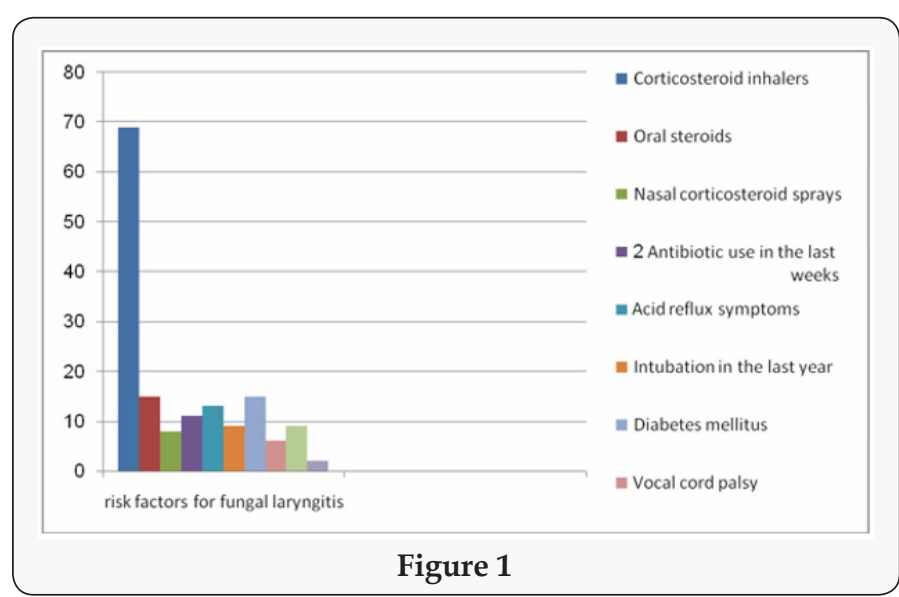

Ninety three patients with the diagnosis of fungal laryngitis were included to our study, thirty four males and fifty nine females, with ages ranging from 17 to 93 (mean=58.74). Six of these patients had a recurrent infection more than three times over a period of five years. Among those patients; 69 patients(74.19\%) were using corticosteroid inhalers, 15 patients(16.13\%) were using oral steroids, 8 patients(8.60\%) were using steroid nasal sprays, 11 patients(11.83\%) were on antibiotics for at least one week during the period of the infection or two week before, 13 patients(13.98\%) were complaining of acid reflux symptoms including heart burn, and throat clearing, 15 patients $(16.13 \%)$ were diabetics, 9 patients(9.68\%) were currently smoking, 38 patients(40.86\%) were ex- smokers, 6 patients(6.45\%) were having vocal cord palsy, 9 patients(9.68\%) were intubated during the last year before infection, 2 patients(2.15\%) were immunocompromised, and the other 91 cases were immunocompetent (Figure 1). Most of those patients presented complaining of Dysphonia alone as the main symptom ( 84 patients $=90.32 \%$ ), see Table 1 for the list of symptoms at presentation. True vocal cords were the most affected laryngeal site with fungal inflammation (92 patients), 5 patients had a defuse infection in all the larynx, 4 patients have the arytenoids affected with the vocal cords, one patient with epiglottic involvement, and one patient with false vocal cord involvement (Table 1). Most of the patients $(27$ patients $=29.03 \%)$ presented with a white thin "faint" plaque like lesion, 16 patients(17.20\%) presented with thick kind of white plaque like lesion, 18 patients(19.35\%) presented with white thick discharge that is not coughed up or cleared, 14 patients(15.05\%) presented with white spots and nodular patterned lesions, 16 patients(17.20\%) presented with white non elevated area of fainting blood vessels and surrounding inflammation and increased vascularity, 2 patients(2.15\%) presented with thick and deep white ulcers (Figure 2). Treatment with antifungal oral agents were enough to cure all the cases but it needed varying time length of treatment ranging from one to twelve weeks, with most of the patients cured after 4 week course of Fluconazole (Figure 3). It represents the period of Fluconazole treatment needed to cure laryngeal candida in our study. Notice that most patients needed 2-4 weeks period of Fluconazole.

Table 1:

\begin{tabular}{|c|c|}
\hline Main Presenting Symptom & Number of Patients \\
\hline Dysphonia & 84 \\
\hline Dysphagia & 4 \\
\hline Difficulty breathing & 4 \\
\hline Cough & 2 \\
\hline Throat pain & 1 \\
\hline
\end{tabular}

types of presenting lesion in fungal laryngitis

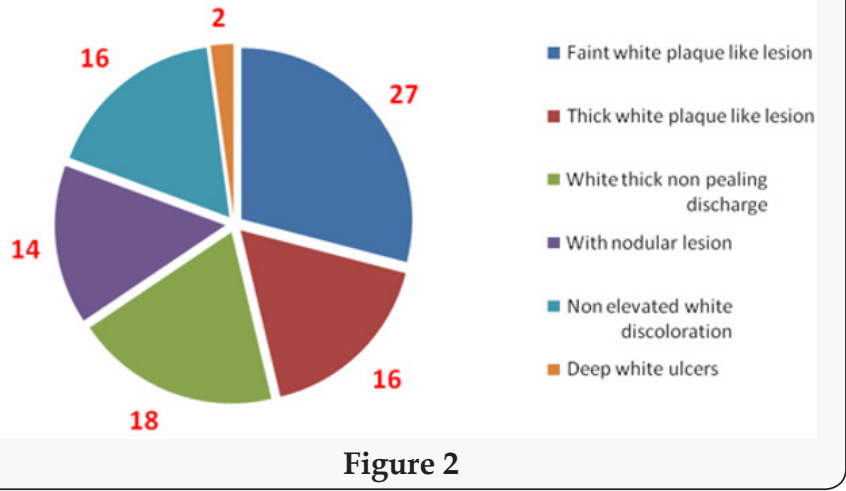




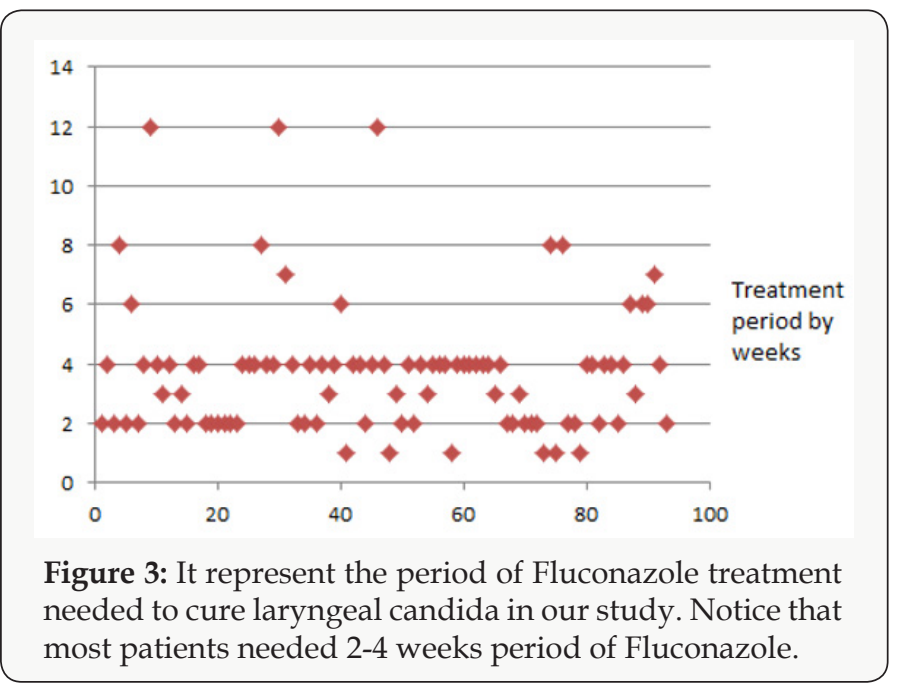

\section{Discussion}

Fungal laryngitis is a rare disease that is known to be seen in immunocompromised patients, nevertheless, this disease is being more often seen in immunocompetent patients recently as well, which might be due to the development of high definition laryngoscopes and increase physician's awareness about this disease with time. We think that fungal laryngitis in immunocompetent patients is still underestimated and frequently missed by otolaryngologists due to many reasons including the varying degrees of presentations the lesion of the fungal infection could present with along the laryngeal mucosa, sometimes subtle faint changes may lead to this diagnosis that might not be noticed in a rapid look at the vocal cord with mirror examination or even laryngoscopes, but could appear obviously in a detailed high definition laryngoscopy especially when the examiner is anticipating it by knowing the risk factors that might be involved and the changes that he/she is looking for. Moreover, we think that other laryngeal problems that have subtle and vague presentation such as laryngopharyngeal reflux (LPR) might be over diagnosed in patients without obviously seen lesion in a way to justify patient's vocal cord dysfunction, leading to label patients with this diagnosis and prescribing them an almost lifelong treatment without much improvement on his/her vocal abilities, while in most of the cases a more real problem such as fungal infection could be seen by a detailed and comprehensive exam and treated with 2-4 weeks of antifungal tablet. This condition is not well studied in the literature, few articles talk about the occurrence of these infections in immunocompetent people, although they are not uncommonly present, the thing that decrease physician's awareness of such a disease and its mode of presentation and progression $[5,6]$. Among the ninety three patients included in this study we found several types of presentation of fungal laryngitis that ranges from an obvious thick white fungal plaque to a very tiny smooth faint white spots that need very detailed exam to notice them. We divided patients into six major groups according to the types of presentation, so it may become easier to other otolaryngologists to notice these changes.

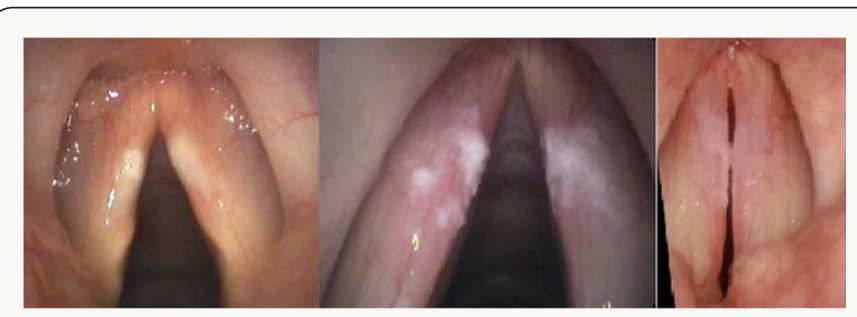

Figure 4: Different degrees of thin faint white plaque like lesions.

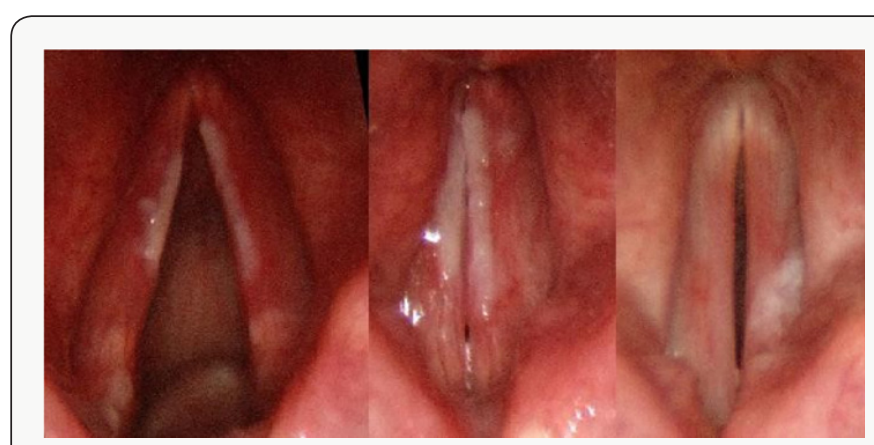

Figure 5: This is the same patient with a thick white plaque like lesion, before treatment (left and middle), and after treatment.

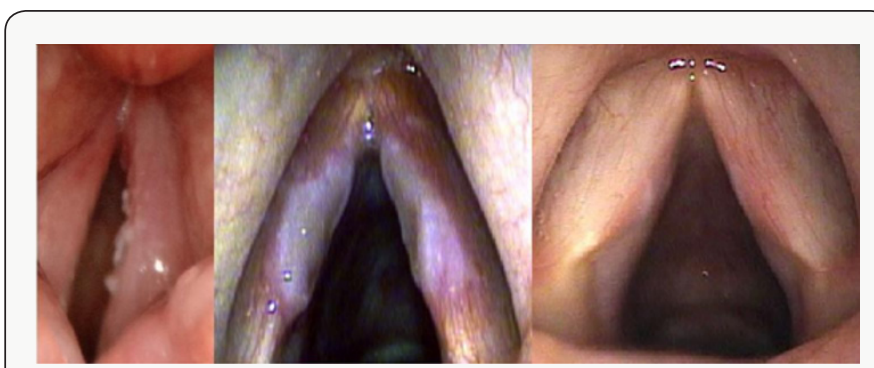

Figure 6: This represent the different appearance between the discharge type (Left), the ulcer type (Middle), and the discoloration type (Right).

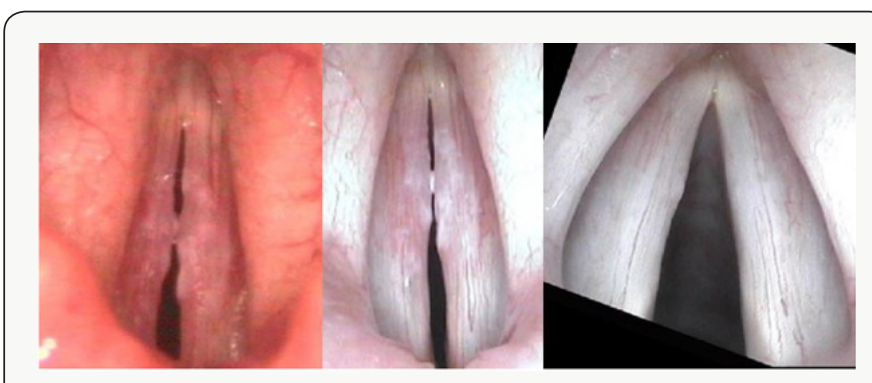

Figure 7: This the same patient with the nodular type lesion (Left), with strobe light on (Middle), and after treatment (Right).

All these lesions were associated with vocal cord stiffness and decrease mucosal wave vibration on stroposcopy, sometimes causing mass effect due to its elevated nature which explains the vocal dysfunction most patients complained of. Most of the patients (27 patients $=29.03 \%$ ) presented with a faint white plaque covering part of the vocal cord edge and surrounded by 
inflammatory reaction causing decrease mucosal wave vibration (Figure 4), sixteen patients presented with a thicker more elevated and more prominent white scar like plaque that could be misdiagnosed as post-surgical scar or tumor growth (Figure 5). Eighteen patients presented with a thick milky white non coughed or cleared discharge that was sticking to specific place over the vocal cord and surrounded by inflammatory changes and vocal cord stiffness. sixteen patients presented with smooth non elevated whitish discoloration with fainting of blood vessels, surrounded by increased vascularity and vocal cord stiffness. Fourteen patients presented with nodular white elevated lesions or flat white spots that adversely affect the vibratory margin of the vocal cord and causing dysphonia. And two patients presented with a severe form of deep and thick ulcer-like white elevated lesion on the vocal cords (Figures 6 \& 7). This the same patient with the nodular type lesion (Left), with strobe light on (Middle), and after treatment (Right).

Obviously, some of those patients needed a detailed and patient exam to make it possible for these lesions to show, because a rapid laryngoscopy that doesn't pay much attention to these simple changes may miss the correct the diagnosis and treat the patient empirically with anti-reflux treatment or speech therapy.

And that is why we studied the previous diagnoses our patients have been labeled of before they presented to us since the time, they started to have symptoms $[7,8]$. This analysis revealed that 17 patients were diagnosed and treated for laryngopharyngeal reflux(LPR), 11 patients were diagnosed to have bacterial infection in the throat, larynx or sinuses and treated with antibiotics, 10 patients were diagnosed with some sort of airway allergy and treated with anti-allergic medications and steroids, 10 patients were diagnosed to have a mass lesion on the vocal cords varying between a polyp, a nodule, and leukoplakia, one patient was diagnosed to have viral ulcer of the vocal cord and treated with antiviral medications. All those patients didn't improve on the treatment prescribed according to these diagnoses and came for second opinion. This clarifies similarities between fungal infection presentation and other more diagnosed diseases and gives and impression that many of these diseases are over-diagnosed and could be more accurately diagnosed when the type of presentation and appearance of fungal laryngitis became more known and popular between otolaryngologists, and when risk factors of this disease are much appreciated. Analysis of presumed risk factors was very important in the sense that it may give a hint to the examiner to be more detailed and to look for the previously described lesions when one or more of these risk factors are present. Among the ninety three patients included in our study just two of them carry the risk of immunocompromised status due to using immunosuppressing medications, while the other ninety one patients were presumably immunocompetent; 96 patients (74.19\%) were using corticosteroid inhalers for other reasons during at least the last week before symptoms, 32 of them have this risk factor as the only risk factor among the factors studied in this research, and six of them has this problem recurrent more than three times over a period of five years of the use of inhalers. Other risk factors studied in this research include the use of oral steroids, the use of nasal steroids, the use of broad spectrum antibiotics in the last 2 weeks before symptoms, others include diabetes mellitus, smoking, radiotherapy or chemotherapy exposure, evidence of acid reflux disease symptoms like heart burn, being intubated in the last year before diagnosis, or having one or two vocal cord palsy that may affect the normal touching between the vocal cord and hence the possible growth of candida. It's important to mention that many patients have multiple risk factors and as (Figure 8) shows just a minority of those patients have only one or two risk factors not combined with the inhaled steroids, while almost half of the patients have inhaled steroid as the only risk factor, this gives an impression that the main factor in those patients' history is using inhaled steroids rather than having diabetes or being smokers for example.
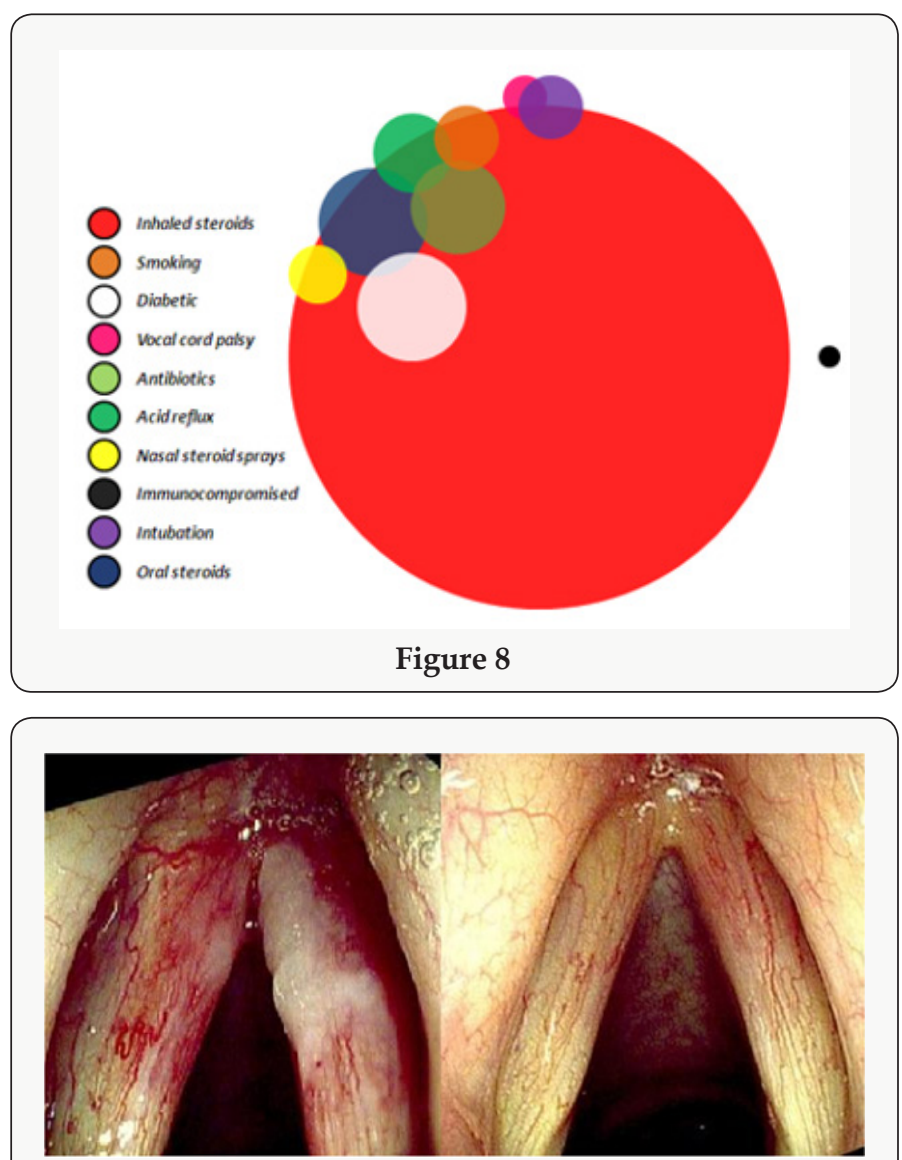

Figure 9: An example of a patient with right vocal cord palsy as the only risk factor of his fungal infection before and after treatment.

Figure 8 Interestingly we found that two of our patients have used only nasal corticosteroid sprays during the two weeks before symptoms, which might increase the awareness of the effect of these sprays use on long term. Four patients among the six with vocal cord palsy had no other risk factors, which might also make us think of the probability of vocal cord irregular or absent movement as a good media for fungus growth (Figure 9). It's important here to say that six of our patients didn't have any of the studied risk 
factors, which makes it important to study this disease more, try to find other causes for it, and makes it clear that this kind of infection is a possible differential diagnosis when such lesions are seen even with the absence of major risk factors. All the cases except one in the study have the true vocal cord involved only or with other structures in the larynx, which explains the presenting complaints of the patients which dysphonia in 84 patients was, other patients presented with cough, throat pain, dysphagia, and breathing problems (Table 2).

Table 2:

\begin{tabular}{|c|c|}
\hline Site of Infection & Number of Patients \\
\hline True vocal cords & 92 \\
\hline False folds & 1 \\
\hline Arytenoids & 4 \\
\hline Epiglottis & 1 \\
\hline All the larynx & 5 \\
\hline
\end{tabular}

While treatment of candida infection in the mouth needs generally 1-2 weeks of oral fluconazole, we found that treatment of laryngeal fungal infections may vary and might be prolonged to 3 months is some sever cases to achieve a complete resolution of the infection and subsequent inflammation which might be related to the fact that most of the patients keep to have the risk factor (the corticosteroid inhaler) along the duration of the disease because stopping it may cause relapse of their respiratory problems. Most patients in our study needed 4 weeks of oral fluconazole to get rid of their infection and go back to normal. The indication of the corticosteroid inhaler was reevaluated, and the need estimated with the prescribing doctor and if patients can't stop using it, they were advised and instructed to do laryngeal water gurgles with Abraham cannula after each inhaler use.

\section{Conclusion}

Fungal laryngitis can present in various forms, the diagnosis is clinical and is based on anticipating the presence of its various presentations after appreciating the major risk factors. Steroid inhalers are the main risk factor that is closely related to the occurrence of this infection. Difficulty of diagnosis conformation, due to the time needed and the invasiveness of the culturing methods of fungus on the larynx made the diagnosis of the cases in the study purely clinical which is considered one of the main defects of this study, that might decrease the threshold of diagnosing fungal laryngitis for patients with subtle nonspecific changes and hence a false diagnosis like the problem we encounter in laryngopharyngeal reflux, for that reason, we recommend constructing a prospective study on a group of patient using inhaled steroid and following them up for 2-3 years with multiple laryngoscopies for any evidence of fungal laryngitis, possibly having a laryngeal brush culture before starting the treatment with antifungal medications, this would be the best way for evaluation of the true incidence of fungal laryngitis in inhaled steroid exposed patients with the ability to estimate the statistical evidence of the relation between the risk factor and the disease. More research is needed on this field; this may increase Doctors awareness about the possibility of this infection in immunocompetent patients [9]. Proper assessment of hoarseness of voice in patients on inhaled steroids and low threshold of laryngoscopic examination is a key point before labeling the patient with another diagnosis like allergy or reflux especially for patients with risk factors.

\section{References}

1. Kevin K Wong, Pia Pace Asciak, Bella Wu, Murray D Morrison (2009) Laryngeal Candidiasis in the Outpatient Setting. Journal of otolaryngology head \& Neck Surgery 38(6): 624-627.

2. Oguz Guclu Burak Ulkumen, Tahir Bulbul (2006) Candida laryngitis. Journal of Otolaryngology- Head and Neck Surgery 135(3): 483-484.

3. Swapna K Chandran, Karen M Lyons, Venu Divi, Matthew Geyer, Robert T Sataloff (2009) Fungal laryngitis. ENT-Ear, Nose \& Throat Journal 88(8):1026.

4. Andrew Mallon DO, Rima A De Fatta, Robert T Sataloff (2012) Acute candidal pharyngolaryngitis. Ear, Nose \& Throat Journal 91(7): 31-32.

5. Hisham M Mehanna, Tony Kuo, John Chaplin, Graeme Taylor, Randall P Marton (2004) Fungal laryngitis in Immunocompetent patients. The Journal of Laryngology \& Otology 118: 379-381.

6. Sulica I (2005) Laryngeal Thrush. Ann Otol Rhinol Laryngol 114(5): 369-375.

7. Gallivan GJ, Gallivan Kl I, Gallivan HK (2007) Inhaled corticosteroids: hazardous effects on voice-an update. J Voice 21(1): 101-111.

8. Balsam D, Sorrano D, Barax C (1992) Candida epiglottitis presenting as stridor in a child with HIV infection. Pediatr Radiol 22(3): 235-236.

9. Tashjian LS, Peacock JE (1984) Laryngeal candidiasis. Report of seven cases and review of the literature. Arch Otolaryngol 110(12): 806-809.

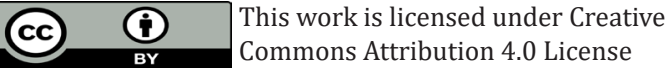

To Submit Your Article Click Here:

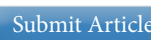

DOI: $10.32474 /$ SJO.2018.01.000114

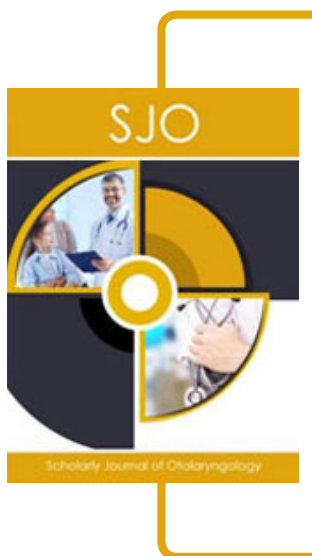

Scholarly Journal of Otolaryngology

\section{Assets of Publishing with us}

- Global archiving of articles

- Immediate, unrestricted online access

- Rigorous Peer Review Process

- Authors Retain Copyrights

- Unique DOI for all articles 